\title{
Play with Me! Gender-Typed Social Play Behavior Analysis in Interactive Tag Games
}

\author{
Alejandro Moreno ${ }^{1}$, Ronald Poppe ${ }^{2}$, and Dirk Heylen ${ }^{1}$ \\ 1 Human Media Interaction, University of Twente, The Netherlands, \\ a.m.morenocelleri@utwente.nl,d.k.j.heylen@utwente.nl \\ 2 Interaction Technology Group, Utrecht University, The Netherlands, \\ r.w.poppe@uu.nl
}

\begin{abstract}
Promoting social behavior is one of the key goals in interactive games. In this paper, we present an experimental study in the Interactive Tag Playground (ITP) to investigate whether social behaviors reported in literature can also be observed through automated analysis. We do this by analyzing players' positions and roles, which the ITP logs automatically. Specifically, we address the effect that gender and age have on the amount of tags and the distance that players keep between them. Our findings largely replicate existing research, although not all hypothesized differences reached a level of statistical significance. With this proof-of-concept study, we have paved the way for the automated analysis of play, which can aid in making interactive playgrounds more engaging.
\end{abstract}

Key words: Interactive Playgrounds, Social Behavior Analysis, Children's Play, Ambient Entertainment

\section{Introduction}

Studies have shown that play is essential for the development of children $[1,2]$, of their physical capabilities [3], cognitive processes [4], and social understanding [5]. Playgrounds, parks, or, in general, spaces that allow children to move and interact with other children freely, have been the typical settings for play. Nowadays, children spend a significant amount of time consuming online digital media, and a considerable part of this time is dedicated to digital gaming [6]. Most young people play video games at least occasionally, and many, especially boys, play them on a daily basis $[7,8]$. The consequences of these changes in children's play habits are increasingly becoming apparent. There is an alarming trend of children playing "together and apart", playing games with others but not directly interacting with them [9].

To counter this trend, interactive technology is being used to design games that aim to bring players closer together and trigger social interactions amongst them [10]. This can be achieved by implementing games where multiple players play together, such as interactive playgrounds [11, 12], or by explicitly designing interactive installations that target social interactions such as competition [13] 
or collaboration [14]. To evaluate whether these games achieve their specified goals, questionnaires, observational studies, and interviews are typically used $[15,16]$. This leaves room for improvement as these measurement techniques are usually subjective and their application in analyzing play behavior is often time-consuming. Considering that there are fundamental differences in social play behavior between boys and girls [17, 18], being able to measure and act upon these differences in-game could help make interactive play more engaging.

This paper addresses this scenario by investigating whether players' social behavior can be objectively measured and analyzed automatically in the Interactive Tag Playground (ITP). The ITP is an interactive game installation that uses sound, sensor and projection technology to enhance the traditional game of tag $[19,20]$. It has been designed to retain the essence of the original game while making it possible to introduce novel gameplay elements easily. The ITP tracks players and displays a colored circle underneath them. The color of the circle indicates the role of the player: orange for taggers, blue for runners. To facilitate the tracking of the players, instead of physically touching other players to tag them, the tagger has to get his circle to overlap with a runner's circle. The ITP can automatically measure cues that can aid in the analysis of social behavior, such as the distance between players or the number of times a player is tagged.

This paper is structured as follows: Section 2 presents literature related to gender-typed behavior, typical behavior exhibited specifically by boys and girls, and how it affects how children play together. In Section 3 we describe our experimental setup and the user study that we conducted to analyze gendertyped behavior. In Section 4 we present and discuss the results of our study. Finally, in Section 5, we summarize our findings.

\section{Age and Gender Effects on Social Play Behavior}

Children prefer to interact with other children of the same gender [21, 22, 23, 24]. This tendency starts very early in childhood, and lasts well until children reach puberty. A study of children between one and twelve years old showed that, although this behavior is already shown at an early age, it is more evident as children grow older [25]. This is due to the fact that they become more conscious of, and grow into, their own gender as time goes by. Moreover, the behavioral patterns exhibited by these groups differ between genders. Boys prefer to interact in larger groups, leading to many "shallow" relationships, whereas girls prefer smaller groups, typically of only a couple of "best" friends [26,21]. Differences in behavior that are typically attributed to gender are called gender-typed behavior.

Gender-typed behavior is not only limited to everyday social interactions, but it can also be observed during play. Boys, for instance, often prefer to play in public spaces such as streets, whereas girls usually get together in private homes or yards [17]. Maccoby and Jacklin showed that boys usually play in groups, whereas girls play mostly with one or two best female friends [18]. Although children most often play with children of the same gender, cross-sex play is 
also seen in children's play. Often this is due to external factors such as limited availability in playing partners [27].

Preference towards certain play activities that children engage in, and the manner in which the activities are carried out, also differs between genders. Pellegrini observed that rough-and-tumble play is not only seen more often in boys than in girls, but it is also related to the social standing amongst boys [28]. Archer also presented several studies where boys engaged in more active play than girls [29]. Eccles and Harold found that gender plays a big part in the attitude of children towards certain sports [30]. Interestingly, they mentioned that the preference was not so much about their aptitude towards the sport itself, but more related to gender-role socialization. In other words, the more they saw sports as being appropriate for their gender, the higher they rated their abilities. Cherney and London considered not only sports, but also toys, computer games, TV shows and outdoor activities differences for kids between five and thirteen years old. They found gender to be a significant factor [31]. Boys preferred to spend more time doing sports, playing video games and watching television, whereas girls preferred only to watch television. Also, the activities they preferred became more gender-typed with age.

Since the ITP automatically collects players' positions and roles, this paper serves as an experimental validation of whether this information can be used to analyze social behavior. We are especially interested in whether gender and age change the way in which players behave during interactive tag games. In the next section, we present how the data obtained from tracking players in the ITP can be used to analyze social behavior automatically during games.

\section{Objective Analysis of Social Behavior in the ITP}

To test if we can measure differences in gender-typed social behavior in the ITP, we conducted a user study with children of different ages and genders playing interactive tag. Each session consisted of four children, two boys and two girls, of the same age group. The playing area was $6 \mathrm{~m} \times 5 \mathrm{~m}$. This arrangement was designed to bring forth differences in play behavior, as seen in the literature. The user study was approved by the university's ethical committee. Our hypotheses and the cues that we measure are explained in Sections 3.1 and 3.2, respectively.

\subsection{Hypotheses and Operationalization}

We define social engagement as the construct to analyze how children's social play behavior differs between genders and age groups. Social engagement refers to how socially active players are during the game. We identified in the literature three different approaches that are normally followed when studying gender-typed social engagement: the analysis of differences between same-gender interactions (i.e. boy-boy versus girl-girl), differences between mixed-gender and same-gender interactions (i.e. boy-girl and girl-boy versus boy-boy and girl-girl), and differences between interactions of children of different ages. 
For same-gender interactions, differences have been observed between boys and girls. Specifically, boys tend to play in larger groups than girls [18], which leads to many, but superficial, relationships $[21,26]$. On the other hand, girls play in smaller groups, which leads to fewer, but more intense, relationships. We believe this will be reflected in the ITP by a) girls interacting with (tagging) girls more often than boys interacting with boys, and b) girls staying closer to other girls than boys stay from other boys. This leads to the first set of hypotheses:

Hypothesis 1a (H1-Tg) The average number of tags between girls is higher than between boys.

Hypothesis 1b (H1-Dt) The average distance between boys is bigger than the distance between girls.

In regards to same-gender versus mixed-gender interactions, researchers have suggested that children usually form groups made up of children of the same gender $[22,24]$, and that children mostly play with children of the same gender [27]. We believe this will lead to a) players preferring to tag players of the same gender over those of the opposite gender, and b) players staying closer to players of the same gender. We thus formulate a second set of hypotheses:

Hypothesis 2a (H2-Tg) Players of the same gender will tag each other at a higher rate than players of the opposite gender will.

Hypothesis 2b (H2-Dt) The average distance between pairs of the same gender is smaller than the distance between pairs of opposite genders.

Finally, gender-typed behavior is exhibited from a very young age up until the teen years. Studies have shown that this type of behavior becomes more evident as children age [25]. We believe that this will manifest itself in the form of greater differences between the measurements of behavioral cues for young and older children. Our final set of hypotheses is as follows:

Hypothesis 3a (H3-Tg) The number of tags between players of the same gender will be higher for the older children.

Hypothesis 3b (H3-Dt) The distance between pairs of the same gender will be smaller for the older children.

\subsection{Behavioral Cues}

To investigate our hypotheses, we use two behavioral cues derived solely from the position and role information of each player. The first cue, $T g$, is the average number of tags between players per session. By analyzing whom a player tags the most, we should be able to find if there is a preference to interact with players based on their genders. The ITP keeps track of the roles of the players and, by counting the number of times the tagger role switches, we can measure the number of times a player has tagged someone. We consider the gender of a player when counting the number of tags. Therefore, we look at the number of tags between male players, female players, and players of the opposite gender. 
The second cue, $D t$, is the average distance between players. By calculating the distance to other players, we expect to find preferences related to whom they want to be close to and, therefore, interact with more. Since the ITP tracks players and logs their position during the game, we calculate $D t$ by averaging, over the entire game duration, the distance between any two given players. This means that, inherently, $D t$ is a pairwise cue. When analyzing this cue, we specifically look at pairs of players, taking into consideration their genders. As such, we analyze the distance between pairs of male players, female players, and players of the opposite gender. $D t$ is measured in meters.

\subsection{Experimental Design}

In this study, we specifically look at how children's social engagement is affected by gender and age. For the first variable, gender, we designed each session in such a way that the same number of players from each gender played together. For our second variable, age, we had players of 6-8 years old playing together (A-Y), and players of 9-10 years old playing together (A-O). We will refer to these two groups as younger and older children, respectively.

Thirty two children from two different schools were invited to our university over the span of two days. The children took part in many activities, including playing tag in the ITP. The children were divided into groups of four players, which led to a total of eight groups. Sixteen of the children were boys, and half of those sixteen were 6-8 years old. This means that we had eight young boys, eight young girls, eight old boys and eight old girls. The consent forms that we used only asked whether we could record and analyze the data of the children. Therefore, there are no pictures or video recordings of the game sessions.

\subsection{Experimental Procedure}

Once the children arrived at the university, they were shown all the possible activities they could partake in. After this, they were allowed to move around and participate in any activity they wished. For each session, two boys and two girls were chosen randomly from the pool of available children. This meant either choosing children who were at the ITP or, if needed, asking children who were at other stations if they wanted to play a game of tag.

Once the children were selected, the game was explained to them. Afterwards, they were asked to stand on specific colored stars located in the corners of the playground (Figure 1). Girls were asked to stand on the red stars, whereas boys were asked to stand on the yellow ones. Using this method, we could know which players were male and which females at the start of each game session. Each group played the game for one minute and a half. After each session, players were asked to participate in a very brief feedback session.

Manual Annotation of Data Although the performance of ITP's tracker is good (on average, two track switches per game session) [32], occasionally, the label assigned to each player by the ITP changes when players run too close 


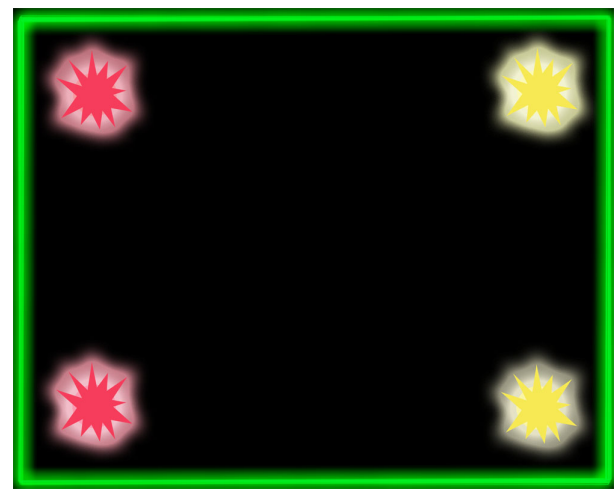

Fig. 1. Projected image before every tag game session in the ITP.

to each other. For this study, we had to ensure that the labels assigned to the players at the beginning of the game were maintained throughout the entire session, as a player's starting position indicates its gender. Therefore, the track information provided by the ITP was manually revised and corrected.

The manual correction was carried out by saving unprocessed depth images from the game sessions. These images were watched next to a visual representation of the ITP's tracker output on a frame-by-frame basis. Although we could not identify players from the depth images, their contours provided sufficient information to distinguish them from each other when in close proximity. Whenever labels were incorrectly assigned, they were corrected. When a player was lost by the tracker, its position was annotated. After the manual correction, we ran a median filter with a window of $1 / 3$ of a second on the position data to smooth it. By manually correcting the label assignment process, we made sure that the analysis of behavior could be carried out not only at the group level but also at the individual level. Nonetheless, this process significantly increases the time needed to analyze behavior.

\section{Experimental Results}

To evaluate social engagement, we study in detail the pairwise interactions between the players. We specifically look at how the distance between pairs of players $(D t)$ and the number of tags $(T g)$ differ between genders and age groups. A total of eight play sessions were analyzed: four play sessions in the A-Y condition, and four in the A-O condition. Each session consisted of two boys playing with two girls in the same age group.

\subsection{Average Number of Tags per Player}

We first calculate the tagging ratio of the players based on their gender. We count the times a player tagged children of a specific gender, and divide this value by the player's total number of tags. Results are shown in Table 1. 
Table 1. Tagging ratio based on a player's gender and age. Male-Male represents tags from a boy to other boys. Female-Female represents tags from a girl to other girls.

\begin{tabular}{|c|c|c|c|}
\hline \multicolumn{4}{|c|}{ Tagging Ratio } \\
\hline & Male-M & ale-Female & Average \\
\hline A-Y & 0.33 & 0.30 & 0.32 \\
\hline $\mathrm{A}-\mathrm{O}$ & 0.37 & 0.44 & 0.41 \\
\hline Average & 0.35 & 0.37 & \\
\hline
\end{tabular}

Considering that every game session consists of two boys and two girls, for any given player, the baseline ratio of tagging a player of the same gender is 0.33 , and 0.67 for a player of the opposite gender. We can see that only the older children show a preference to tag other players of the same gender (0.41). In the A-Y condition, we can see that the tagging ratio between boys is 0.33 , which means there is no preference. For the girls, this value is 0.30 , which is also close to the baseline value. However, when we look at the A-O condition, we can see that the tagging ratio for boys increases to 0.37 , and the ratio of girls tagging other girls increases to 0.44 .

To get a better picture of how tagging behavior changes between conditions, we also calculate the number of tags between players. Since there are two players of each gender, we normalize the number of tags between players of opposite genders by dividing them by two. Results are shown in Table 2 .

Table 2. Average number of tags between players based on their gender and age. The number of tags between players of opposite genders has been normalized.

\begin{tabular}{ccccc}
\multicolumn{4}{c}{ Normalized Average Number of Tags } & Between Players per Session \\
\hline & Male-Male & Male-Female Female-Female & Female-Male \\
\hline A-Y & 1.75 & 1.81 & 1.63 & 1.81 \\
A-O & 1.88 & 1.50 & 2.25 & 1.56 \\
\hline Average & 1.81 & 1.66 & 1.94 & 1.69
\end{tabular}

The average number of tags between girls (1.63) is lower than for boys (1.75) when considering the A-Y condition, but in the A-O condition the average number of tags per player for girls (2.25) is higher than for boys (1.88). This means that, apparently, the age of the children has an influence on tagging preferences. Also, it seems that girls prefer to interact with girls more often than boys do with boys, at least for the older group of children.

The average number of tags between players of the same gender increases in the A-O condition when compared to the A-Y condition. Consequently, the average number of tags between players of the opposite gender decreases. The difference is more marked for girls, which changes from -0.18 (1.63-1.81) to 0.69 (2.25-1.56). For boys, the difference in tagging preference from A-Y to A-O changes from -0.06 (1.75-1.81) to 0.38 (1.88-1.50).

The interaction between $T g$, gender and age can be seen in Figure 2. The graph shows an important increase in the number of tags between girls when 


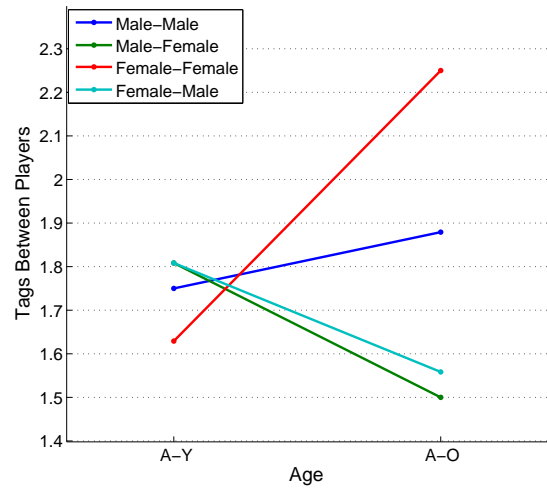

Fig. 2. Average number of tags between players based on age and gender.

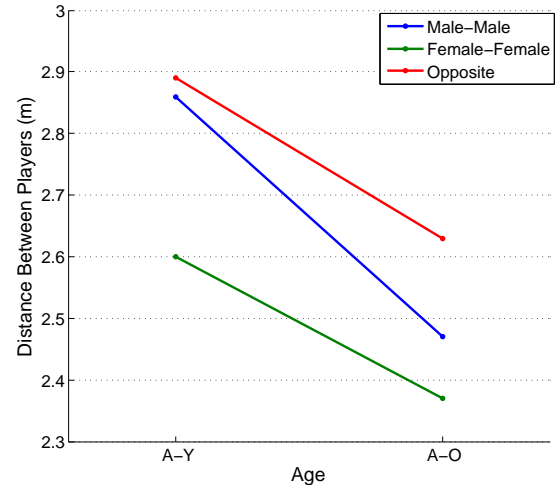

Fig. 3. Average distance between players based on age and gender.

going from the A-Y to the A-O condition. There is also an increase in the number of tags between boys, but it is considerably smaller than the one seen for girls. The number of tags between players of opposite genders decreases in the A-O condition. Notice that the younger girls have the lowest number of tags between players of the same gender, but the older girls have the most.

To evaluate H1-Tg and H3-Tg, we run a 2-way factorial ANOVA to find the effect of gender and age on the number of tags between players. There was no statistically significant interaction between the effects of gender and age on the number of tags per player $(F(1,28)=0.415, p=n s)$. To assess H2-Tg, we run a set of 1-sample t-tests against the baseline tagging ratio for players of the same gender (0.33). We found no statistically significant differences in any of the four tests (number of tags between males and number tags between females for the younger and older children).

\subsection{Average Distance to Other Players}

In Table 3 we can see that for both the A-Y and A-O conditions, the distance that girls keep between them is shorter than the distance boys keep. When looking at A-Y, we can see that the distance between boys $(2.86 \mathrm{~m})$ is larger than the distance between girls $(2.60 \mathrm{~m})$, but is almost the same as the distance between players of opposite genders $(2.89 \mathrm{~m})$. This means that boys did not show a marked preference in staying close to players of the same gender. Girls, on the other hand, already show gender-typed behavior in the young group.

Table 3. Average distance to other players based on their gender and age.

\begin{tabular}{cccc}
\multicolumn{4}{c}{ Average Distance to other Players $(\mathbf{m})$} \\
\hline \multicolumn{4}{c}{ Male-Male Female-Female Opposite Gender } \\
\hline A-Y & 2.86 & 2.60 & 2.89 \\
A-O & 2.47 & 2.37 & 2.63 \\
\hline Average & 2.67 & 2.49 & 2.76
\end{tabular}


When looking at the A-O sessions, the distances between all the players shrink, and boys start to exhibit a preference to stay close to other boys. This can be seen in the $2.47 \mathrm{~m}$ they keep from each other, in comparison to the 2.63 $\mathrm{m}$ that players keep to players of the opposite gender. Girls do not really change their behavior, and prefer to stay even closer to other girls $(2.37 \mathrm{~m})$. The graph with the interactions between the variables can be seen in Figure 3.

To test H1-Dt, H2-Dt and H3-Dt, we conducted a 2-way factorial ANOVA to measure the effect of gender and age on the distance between players. The test shows a statistically significant decrease in $D t$ for the older children $(F(1,42)=$ $11.78, p<0.05)$. We also found that gender has a statistically significant effect on $D t(F(2,42)=4.215, p<0.05)$. After running a post-hoc test on gender, we found that the distance between girls was significantly lower than the distance between players of different gender $(p<0.05)$. However, there was no statistical difference in $D t$ between boys and girls, nor for boys and opposite gender pairs. Finally, the interaction between age and gender did not show a statistically significant effect on $D t(F(2,42)=0.275, p=n s)$.

\subsection{Discussion of Social Engagement Results}

The results show that there are some differences in social engagement with respect to gender and age in the ITP. For the number of tags between players, $\mathrm{Tg}$, we only found small differences. From these differences, the most noticeable one was found for girls in the A-O condition, which had the highest number of tags between them when compared to tags between boys or between mixed gender pairs. Also, the difference in tagging behavior between the girls in the younger group and the older group was the most pronounced. Nonetheless, neither the 2-way factorial ANOVA nor the 1-sample t-tests we ran showed statistically significant differences in tagging ratios or number of tags per player. Consequently, we reject $\mathrm{H} 1-\mathrm{Tg}, \mathrm{H} 2-\mathrm{Tg}$ and $\mathrm{H} 3-\mathrm{Tg}$.

When looking at the distance between players, $D t$, we found the largest differences for girls in the A-O condition. In this case, the older girls kept the shortest distance between them when compared to the distance between boys or players of opposite genders. The biggest difference between age groups was seen for the boys. We found a statistically significant difference in the distance between girls in comparison to the distance between players of different genders. However, since there was no significant difference between girls and boys, or boys and pairs of children of opposite genders, we reject H1-Dt and H2-Dt. In regards to H3-Dt, we found a statistically significant decrease in the distance between players when going from A-Y to A-O, which confirms this hypothesis.

One possible limitation in our current study is that, by explicitly telling boys and girls to stand on specific corners to start the game, we may be priming them into thinking about their gender and enhancing their awareness of it. Moreover, studies suggest that gender segregation is more evident when situations have not been structured by adults [17], which could mean that by forcibly arranging mixed gender play groups, children may feel pressured to adapt their playstyle 
to fit the situation. We have no evidence to suggest that either happened, but it would be better if the children themselves chose who they wanted to play with.

This relates to another limitation in our experiment: the number of children that took part in the experiment. Since we only had eight mixed gender sessions, the number of observations was quite low. This could be one of the reasons why some of the observed differences were not found to be statistically significant. With a larger sample size, the variance of the measurements may diminish.

Lastly, since the children that played together knew each other, we may be overlooking the effect that their social relationships have on their playing preferences. Since each group was composed of randomly selected children, this effect might be mitigated. Nonetheless, future studies could be carried out with children that do not know each other to see if the behavior changes.

\section{Conclusions}

In this paper, we have addressed the automated analysis of gender-typed social play behavior in the ITP. We have conducted a user study with children of different age groups and genders playing interactive tag. We have analyzed these play sessions using two behavioral cues that were measured unobtrusively by the ITP: the number of tags and the distance between players. Our results generally follow what we expected based on the existing literature on children's play. Our analyses show that age has an effect on the distance that players keep to each other during interactive tag. More specifically, older children tend to stay closer to each other when compared to younger children. We also found that the distance between girls is significantly shorter than the distance between players of opposite genders. Other measurements hint at the presence of gender-typed social play behavior, but the differences were not statistically significant.

These results convey that certain gender-typed behavior is exhibited during interactive tag games, and that it can be measured in the ITP. This is important because it allows us to evaluate game interventions that target specific aspects of social play. For instance, a game intervention that encourages isolated players to interact with other children could be evaluated based on the distance between

players. An intervention to steer passive players to engage other players could be evaluated based on the number of times the player tags others. Although these interventions and the results of this study might be specific to this particular type of game, we consider this study a first step towards the automated analysis of social behavior in interactive playgrounds.

\section{Acknowledgments}

This publication was supported by the Dutch national program COMMIT. 
Gender-Typed Social Play Behavior Analysis in Interactive Tag Games

\section{References}

1. L. A. Barnett, "Developmental benefits of play for children," Journal of Leisure Research, vol. 22, no. 2, pp. 138-153, 1990.

2. A. D. Pellegrini, The Role of Play in Human Development. Oxford University Press, 2009.

3. A. D. Pellegrini and P. K. Smith, "Physical activity play: The nature and function of a neglected aspect of play," Child Development, vol. 69, no. 3, pp. 577-598, 1998.

4. B. Sutton-Smith, Play and learning. New York:Gardner Press, 1979.

5. J. F. Christie and E. Johnsen, "The role of play in social-intellectual development," Review of Educational Research, vol. 53, no. 1, pp. 93-115, 1983.

6. F. Blumberg, M. Blades, and C. Oates, "Youth and new media," Zeitschrift für Psychologie, vol. 221, no. 2, pp. 67-71, 2013.

7. R. A. Desai, S. Krishnan-Sarin, D. Cavallo, and M. N. Potenza, "Video-gaming among high school students: Health correlates, gender differences, and problematic gaming," Pediatrics, vol. 126, no. 6, pp. 1414-1424, 2010.

8. C. J. Ferguson and C. K. Olson, "Friends, fun, frustration and fantasy: Child motivations for video game play," Motivation and Emotion, vol. 37, no. 1, pp. 154$164,2013$.

9. S. Turkle, Alone Together: Why We Expect More from Technology and Less from Each Other. Basic Books, 2011.

10. R. Courtney and L. L. Scarlatos, "Mind reader: Designing for more intimate social play in video games," in Extended Abstracts of the Conference on Human Factors in Computing Systems, pp. 1211-1216, 2015.

11. A. Moreno, R. van Delden, R. Poppe, and D. Reidsma, "Socially aware interactive playgrounds," IEEE Pervasive Computing, vol. 12, no. 3, pp. 40-47, 2013.

12. R. Poppe, R. van Delden, A. Moreno, and D. Reidsma, "Interactive playgrounds for children," in Playful User Interfaces (A. Nijholt, ed.), pp. 99-118, Springer Verlag, 2014.

13. M. H. Fogtmann, K. Grønbæk, and M. K. Ludvigsen, "Interaction technology for collective and psychomotor training in sports," in Proceedings of the International Conference on Advances in Computer Entertainment Technology, pp. 13:1-13:8, 2011.

14. N. Parés, J. Durany, and A. Carreras, "Massive flux design for an interactive water installation: Water Games," in Proceedings of the International Conference on Advances in Computer Entertainment Technology, pp. 266-269, 2005.

15. S. Bakker, P. Markopoulos, and Y. de Kort, "OPOS: An observation scheme for evaluating head-up play," in Proceedings of the Nordic Conference on HumanComputer Interaction, pp. 33-42, 2008.

16. A. E. Staiano and S. L. Calvert, "The promise of exergames as tools to measure physical health," Entertainment Computing, vol. 2, no. 1, pp. 17 - 21, 2011.

17. E. E. Maccoby, "Gender and relationships: A developmental account," American psychologist, vol. 45, no. 4, pp. 513-520, 1990.

18. E. E. Maccoby and C. N. Jacklin, The psychology of sex differences. Stanford University Press, 1974.

19. A. Moreno, R. van Delden, R. Poppe, D. Reidsma, and D. Heylen, "Augmenting traditional playground games to enhance game experience," in Proceedings of the International Conference on Intelligent Technologies for Interactive Entertainment, pp. 140-149, 2015. 
20. A. Moreno, R. van Delden, R. Poppe, D. Reidsma, and D. Heylen, "Augmenting playspaces to enhance the game experience: A tag game case study," Entertainment Computing, In press.

21. D. Eder and M. T. Hallinan, "Sex differences in children's friendships," American Sociological Review, vol. 43, no. 2, pp. 237-250, 1978.

22. B. Thorne, "Girls and boys together... but mostly apart: Gender arrangements in elementary schools," in Education and Gender Equality (J. Wrigley, ed.), pp. 117132, The Falmer Press, 1992.

23. E. E. Maccoby, The two sexes: Growing up apart, coming together. Harvard University Press, 1998.

24. J. Archer and B. Lloyd, Sex and gender. Cambridge University Press, 2002.

25. S. Ellis, B. Rogoff, and C. C. Cromer, "Age segregation in children's social interactions," Developmental Psychology, vol. 17, no. 4, pp. 399-407, 1981.

26. M. F. Waldrop and C. F. Halverson Jr, "Intensive and extensive peer behavior: Longitudinal and cross-sectional analyses," Child Development, vol. 46, no. 1, pp. 1926, 1975.

27. E. E. Maccoby and C. N. Jacklin, "Gender segregation in childhood," in Advances in Child Development and Behavior (H. W. Reese, ed.), vol. 20, pp. 239-287, JAI, 1987.

28. A. D. Pellegrini, "Boys' rough-and-tumble play, social competence and group composition," British Journal of Developmental Psychology, vol. 11, no. 3, pp. 237-248, 1993.

29. J. Archer, "Childhood gender roles: Social context and organization," in Childhood social development: Contemporary perspectives (H. McGurk, ed.), pp. 31-61, Lawrence Erlbaum Associates Ltd, 1992.

30. J. S. Eccles and R. D. Harold, "Gender differences in sport involvement: Applying the eccles' expectancy-value model," Journal of applied sport psychology, vol. 3, no. 1, pp. 7-35, 1991.

31. I. D. Cherney and K. London, "Gender-linked differences in the toys, television shows, computer games, and outdoor activities of 5-to 13-year-old children," Sex Roles, vol. 54, no. 9-10, pp. 717-726, 2006.

32. A. Moreno and R. Poppe, "Automatic behavior analysis in tag games: From traditional spaces to interactive playgrounds," Journal on Multimodal User Interfaces, vol. 10 , no. 1, pp. 63-75, 2016. 